\title{
Analyse des lacunes visant à évaluer le niveau de préparation des Canadiens à l'introduction des vaccins contre le virus respiratoire syncytial : compte rendu d'une retraite d'experts
}

April Killikelly', Amanda Shane ${ }^{1}$, Man Wah Yeung ${ }^{1}$, Matthew Tunis ${ }^{1}$, Christina Bancej', Althea House', Wendy Vaudry², Dorothy Moore ${ }^{3}$, Caroline Quach ${ }^{4}$

\section{Résumé}

Le virus respiratoire syncytial (VRS) peut provoquer des maladies graves chez les nourrissons et les personnes âgées. Plusieurs candidats vaccins sont en cours de développement et leur utilisation pourrait être autorisée au Canada dans les deux à cinq prochaines années. L'Agence de la santé publique du Canada s'est employée à améliorer le niveau de préparation à l'introduction du vaccin contre le VRS et de candidats vaccins pour l'immunisation passive en organisant une retraite d'experts, dont l'objectif consistait à cerner les lacunes dans les connaissances en matière de surveillance et de recherche et développement, et ce, sous l'angle des priorités de santé publique des provinces et des territoires concernant le VRS.

Nous avons déterminé que les candidats vaccins contre le VRS en cours de développement répondaient pleinement à quatre des cinq priorités de santé publique, et avons mis en évidence les lacunes à combler dans les données concernant l'efficacité potentielle et l'efficacité réelle des vaccins. Nous avons constaté que les données de surveillance disponibles étaient limitées ou suffisantes pour étayer le processus décisionnel relatif à quatre des cinq priorités de santé publique liées au VRS, et avons mis en évidence des lacunes dans les données concernant plusieurs populations clés : (i) pour les cas de VRS de moins de 17 ans, il demeure des lacunes dans les données utilisées en guise de dénominateur dans le calcul de I'incidence et dans les données sur les consultations externes avec soins médicaux; (ii) pour les cas de VRS dans les collectivités autochtones et éloignées, il demeure des lacunes dans les données sur l'incidence, la prévalence, les facteurs de risque particuliers, la faisabilité et I'acceptabilité; (iii) pour les cas de VRS chez les personnes âgées, il demeure des lacunes dans les données sur l'incidence. Ce processus a contribué à démontrer la faisabilité de l'analyse des lacunes dans les données de surveillance pour étayer les décisions relatives aux vaccins et aux produits immunitaires candidats, et a mis en évidence le soutien des intervenants à cet égard.
Cette oeuvre est mise à la disposition selon les termes de la licence internationale Creative Commons Attribution 4.0

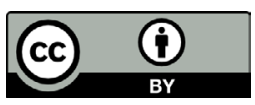

Affiliations

${ }^{1}$ Centre de l'immunisation et des maladies respiratoires infectieuses, Agence de la santé publique du Canada,

Ottawa, ON

${ }^{2}$ Hôpital pour enfants Stollery, Université d'Alberta,

Edmonton, $\mathrm{AB}$

${ }^{3}$ Université McGill, Montréal, QC

${ }^{4}$ Centre Hospitalier Universitaire Sainte-Justine, Université de Montréal, Montréal, QC

${ }^{\star}$ Correspondance : phac.naci-ccni.aspc@canada.ca

Citation proposée : Killikelly A, Shane A, Yeung MW, Tunis M, Bancej C, House A, Vaudry W, Moore D, Quach C. Analyse des lacunes visant à évaluer le niveau de préparation des Canadiens à l'introduction des vaccins contre le virus respiratoire syncytial : compte rendu d'une retraite d'experts. Relevé des maladies transmissibles au Canada 2020;46(4):71-8. https://doi.org/10.14745/ccdr.v46i04a02f

Mots-clés : vaccin, Comité consultatif national de l'immunisation, $\mathrm{CCNI}$, immunisation, VRS, virus respiratoire syncytial

\section{Introduction}

Le Comité consultatif national de l'immunisation (CCNI) est le Groupe technique consultatif national sur la vaccination (GTCNV) du Canada, et a pour mandat de présenter à l'Agence de la santé publique du Canada (ASPC) des recommandations techniques sur l'utilisation des vaccins. Pour formuler ses recommandations, le CCNI s'appuie sur un large éventail de données probantes, notamment les caractéristiques des vaccins, le fardeau de morbidité, ainsi que l'éthique, l'équité, la faisabilité et l'acceptabilité des programmes d'immunisation. Tous ces éléments sont considérés comme des facteurs essentiels pour les décideurs provinciaux et territoriaux (PT) du Canada (1). 
Étant donné que de nombreux vaccins contre le virus respiratoire syncytial (VRS) sont en cours de développement clinique, le $\mathrm{CCNI}$ a besoin de données sur l'épidémiologie du VRS et le coût des nouveaux candidats vaccins pour élaborer des recommandations complètes sur ces facteurs de décision. Compte tenu des besoins de santé publique et de la brièveté des délais impartis pour la formulation des recommandations sur les nouveaux vaccins, I'ASPC a saisi l'occasion d'améliorer le niveau de préparation à l'introduction des vaccins contre le VRS en organisant une retraite d'experts pour analyser les lacunes et mieux comprendre les besoins actuels et futurs des décideurs en matière de vaccination. Le secrétariat du CCNI, appuyé par un comité consultatif technique, a (i) coordonné la détermination des priorités de santé publique (PSP) des PT relativement au VRS; (ii) sélectionné les participants en fonction de leur expertise dans les domaines de la surveillance du VRS, de la recherche, de l'économie, de la santé pédiatrique et de l'immunisation, ainsi que de manière à garantir la représentation des principaux groupes à l'intérieur et à l'extérieur du gouvernement (se reporter à l'annexe 1); et (iii) sélectionné les fabricants de l'industrie pour faire le point sur le progrès des essais cliniques avancés chez les humains (phase 2 ou plus). Les représentants de l'industrie ont été invités à présenter leurs données par téléconférence et les participants à la retraite ont pris part à des discussions confidentielles.

\section{Contexte}

Le fardeau de morbidité lié à l'infection par le VRS pèse lourdement sur le Canada et dans le monde. La répartition par âge de ce fardeau est bimodale, I'incidence la plus forte s'observant au cours des deux premières années de vie et chez les personnes âgées. Le Canada ne dispose pas de données publiées, mais les données d'autres pays à revenu élevé montrent une tendance bimodale des taux d'hospitalisation associés au VRS par tranche de 1000 personnes : 26,3 (0 à 5 mois); 11,3 (6 à 11 mois); 1,4 (12 à 59 mois); 1.0 (65 ans ou plus) $(2,3)$.

En fonction de la population concernée, les vaccins destinés aux populations maternelles, infantiles ou âgées ont des effets différents sur la prévention du VRS. Pour effectuer une analyse des lacunes, nous devions, dans un premier temps, déterminer et comprendre les priorités de santé publique (PSP) liées à la prévention du VRS. Tandis que l'approvisionnement en vaccins est assuré à l'échelon fédéral, le processus décisionnel visant les programmes de vaccination et leur mise en œuvre relèvent de la responsabilité des PT. Pour nous assurer de la pertinence et de l'utilité de notre analyse pour les programmes canadiens d'immunisation, nous avons veillé à garantir la concordance avec les priorités de santé publique des PT. En concertation avec les membres FPT du Comité canadien sur l'immunisation et le Conseil des médecins hygiénistes en chef, nous avons défini les priorités de santé publique PT suivantes pour les programmes de vaccination contre le VRS :

1. PSP-1 : Prévenir I'hospitalisation et le décès des nourrissons

2. PSP-2 : Prévenir I'hospitalisation et le décès des nourrissons à risque élevé

3. PSP-3 : Prévenir l'infection des enfants qui résident dans les régions éloignées

4. PSP-4 : Prévenir l'hospitalisation et le décès des enfants

5. PSP-5 : Prévenir l'hospitalisation et le décès des personnes âgées

Ces PSP liées au VRS ont permis d'orienter l'analyse subséquente des lacunes et d'élaborer des recommandations vaccinales adaptées aux besoins des PT.

Cet article a pour but de résumer les discussions des experts en deux thématiques pour mieux comprendre le niveau de préparation des Canadiens à l'introduction des vaccins contre le VRS : (i) recherche et développement de candidats vaccins et (ii) surveillance actuelle de la santé publique. Pour chacune de ces thématiques, la concordance avec les PSP a été examinée et une analyse des lacunes a été effectuée. Ce processus vise à présenter le raisonnement des experts justifiant la mise en place d'un système renforcé de recherche et de surveillance pour améliorer le niveau de préparation des Canadiens à l'introduction des vaccins contre le VRS.

\section{Résultats clés : recherche et développement de candidats vaccins contre le VRS}

\section{Concordance entre les candidats vaccins et les priorités de santé publique}

Pour assurer la concordance entre les candidats vaccins contre le VRS en développement et les priorités de santé publique des PT, nous avons pris en compte les différents résultats des PSP : la prévention de l'infection par rapport à la prévention de I'hospitalisation ou du décès.

Différentes stratégies de développement de candidats vaccins stimulent les réactions immunologiques protectrices dans le but de prévenir l'infection, la maladie grave ou la transmission (4). Pour formuler des recommandations vaccinales, il est essentiel de faire la distinction entre ces trois résultats. Les données démontrant la capacité d'un vaccin à prévenir la maladie grave ou le décès peuvent former la base d'une recommandation destinée à certaines catégories de personnes (p. ex. cliniciens, sujets vaccinés). Les données démontrant la capacité d'un vaccin à prévenir l'infection ou sa transmission peuvent former la base d'une recommandation destinée à l'ensemble de la population. Ces deux types de recommandations pourraient faire l'objet 
de priorités et de mécanismes de financement très différents dans les programmes de vaccination des PT, ce qui aurait une incidence sur les personnes ayant accès à ces vaccins et le moment où elles peuvent y accéder.

Il existe une solide base de données probantes démontrant que les vaccins qui entraînent la production d'anticorps protecteurs (y compris les vaccins sous-unitaires, les vaccins à particules et les anticorps monoclonaux) préviennent la maladie grave chez les nourrissons. Quant à ceux qui stimulent les réactions cellulaires (notamment les vaccins vivants atténués et les vaccins vectorisés), ils peuvent réduire le risque de maladie grave et de transmission virale grâce aux mécanismes de stimulation liés à la clairance et I'élimination virales induites par les CD8.

Une recommandation vaccinale destinée à l'ensemble de la population est étayée par une base de données probantes démontrant que les réactions humorales et cellulaires protectrices préviennent l'infection ou la transmission au sein de la population. Autrement, il peut être raisonnable de prendre en compte les données probantes indirectes relatives à d'autres vaccins ayant exercé un effet inattendu sur la transmission à l'échelle de la population. Par exemple, le vaccin antirotavirus est indiqué dans la prévention de la gastroentérite aigüe chez les enfants de moins de deux ans. Néanmoins, depuis que le vaccin a été introduit, les taux d'infection à rotavirus chez les enfants et les adultes non vaccinés ont également diminué de manière considérable (5).

Nous avons déterminé que les candidats vaccins en cours de développement répondaient pleinement à quatre des cinq priorités de santé publique des PT (se reporter au tableau 1).

\section{Tableau 1 : Analyse des lacunes des candidats vaccins}

\begin{tabular}{|c|c|c|}
\hline PSP & $\begin{array}{c}\text { PSP provinciales et } \\
\text { territoriales liées } \\
\text { au VRS }\end{array}$ & $\begin{array}{l}\text { Candidat vaccin contre le VRS } \\
\text { en cours de développement } \\
\text { pour répondre à la priorité }\end{array}$ \\
\hline 1 & $\begin{array}{l}\text { Prévenir } \\
\text { I'hospitalisation et le } \\
\text { décès des nourrissons }\end{array}$ & $\begin{array}{l}\text { Janssen (enfants), GSK (populations } \\
\text { maternelles et pédiatriques), } \\
\text { Novavax (populations maternelles), } \\
\text { AstraZeneca/Sanofi Pasteur }\end{array}$ \\
\hline 2 & $\begin{array}{l}\text { Prévenir } \\
\text { I'hospitalisation et le } \\
\text { décès des nourrissons } \\
\text { à risque élevé }\end{array}$ & $\begin{array}{l}\text { Janssen (enfants), GSK (populations } \\
\text { maternelles et pédiatriques), } \\
\text { Novavax (populations maternelles), } \\
\text { AstraZeneca/Sanofi Pasteur }{ }^{a}\end{array}$ \\
\hline 3 & $\begin{array}{l}\text { Prévenir l'infection des } \\
\text { enfants qui résident } \\
\text { dans les régions } \\
\text { éloignées }\end{array}$ & AstraZeneca/Sanofi Pasteur \\
\hline 4 & $\begin{array}{l}\text { Prévenir } \\
\text { l'hospitalisation et le } \\
\text { décès des enfants }\end{array}$ & AstraZeneca/Sanofi Pasteura \\
\hline 5 & $\begin{array}{l}\text { Prévenir } \\
\text { l'hospitalisation et le } \\
\text { décès des personnes } \\
\text { âgées }\end{array}$ & $\begin{array}{l}\text { Janssen (personnes âgées), GSK } \\
\text { (personnes âgées), Novavax } \\
\text { (personnes âgées), Bavarian Nordic }{ }^{a}\end{array}$ \\
\hline
\end{tabular}

Abréviations : PSP, priorité de santé publique; VRS, virus respiratoire syncytial

Le candidat vaccin contre le VRS répond pleinement à la priorité de santé publique

b Le candidat vaccin contre le VRS répond partiellement à la priorité de santé publique
Prévenir l'hospitalisation et le décès des nourrissons (PSP-1), des nourrissons à risque élevé (PSP-2) et des enfants (PSP-4). Janssen, GSK et Novavax développent actuellement des candidats vaccins destinés aux populations infantiles par administration directe ou par immunisation maternelle. AstraZeneca/Sanofi Pasteur développe actuellement un anticorps monoclonal pour lutter contre le fardeau de morbidité du VRS chez les nourrissons qui présentent un risque élevé de maladie grave. Ces candidats vaccins sont entrés dans les phases 2 et 3 du développement clinique pour les nourrissons en bonne santé; les nourrissons à risque élevé en raison de comorbidités; et les enfants en bonne santé. Ces trois priorités de santé publique sont directement ciblées par plusieurs candidats vaccins en phase finale de développement clinique.

\section{Prévenir l'infection des enfants qui résident dans les} régions éloignées (PSP-3). À la fois les vaccins et les produits immunitaires ciblant le VRS pourraient répondre à cette priorité de santé publique. Le palivizumab est le seul produit actuellement disponible. II est uniquement administré aux nourrissons qui présentent un risque élevé de maladie grave induite par le VRS ou, dans le cadre d'un projet pilote particulier mené à Nunavik, à tous les nourrissons à terme qui résident dans les collectivités éloignées du Nord (6). Le palivizumab exerce son activité pendant environ un mois. Il se peut que la prochaine génération de produits d'anticorps monoclonaux, notamment le produit actuellement développé par AstraZeneca/Sanofi Pasteur, ait une période d'efficacité plus longue (potentiellement supérieure à cinq mois). Cela pourrait accroître la facilité d'utilisation de ces produits dans les régions où les systèmes de santé sont difficiles d'accès, notamment les collectivités éloignées.

Le palivizumab et les autres anticorps monoclonaux devraient prévenir la maladie grave induite par le VRS, mais on ignore si ces produits peuvent également réduire le risque d'infection par le VRS. Des recherches complémentaires sont nécessaires sur la faisabilité et l'acceptabilité des programmes de vaccination contre le VRS dans les collectivités éloignées du Canada. Des travaux sont en cours à Nunavik, dans le nord du Québec, pour évaluer l'incidence d'un élargissement de l'accès au palivizumab à tous les nourrissons de moins de trois mois (6). Bien que les données issues de cette étude contribueront à étayer la prise de décisions concernant l'utilisation multidose du palivizumab dans les collectivités nordiques et éloignées, elles pourraient ne pas s'appliquer directement aux nouveaux anticorps monoclonaux du fait de l'augmentation potentielle de la durée de protection. Des études complémentaires pourraient être nécessaires pour répondre pleinement à cette priorité de santé publique.

\section{Prévenir l'hospitalisation et le décès des personnes} âgées (PSP-5). Janssen, GSK, Novavax et Bavarian Nordic développent actuellement des vaccins destinés aux personnes âgées et notamment à celles souffrant de comorbidités (la bronchopneumopathie chronique obstructive, entre autres). Cette priorité de santé publique est directement ciblée par 
plusieurs candidats vaccins en phase finale de développement clinique. En outre, cette population pourrait être protégée par l'immunisation des cohortes plus récentes (immunité collective), comme pour le rotavirus ou l'infection pneumococcique (5).

\section{Lacunes cernées concernant la recherche et le développement des candidats vaccins}

Outre l'analyse des lacunes concernant les priorités des PT et les candidats vaccins, les fabricants développant un vaccin ou un produit immunitaire ciblant le VRS entré dans les dernières phases des programmes d'essais cliniques ont été invités à présenter leurs données sur l'efficacité réelle, l'innocuité et l'immunogénicité du vaccin, ainsi que sur d'autres sujets pertinents (4). À la lumière de ces échanges confidentiels, nous avons cerné les points sur lesquels des recherches ou des analyses complémentaires seraient nécessaires pour bien comprendre l'efficacité potentielle et l'efficacité réelle des candidats vaccins en développement.

I. Administration concomitante de vaccins contre le VRS. Les antigènes présentés par plusieurs candidats vaccins pourraient être identiques à ceux ciblés par les anticorps monoclonaux anti-VRS, dont le palivizumab. L'innocuité et l'efficacité d'une administration concomitante de vaccins et d'anticorps monoclonaux ciblant le VRS n'ont pas été évaluées.

II. Cohérence des définitions de cas. Les définitions de cas des maladies associées au VRS varient d'un essai clinique à I'autre. Cela peut limiter les comparaisons directes entre les données nécessaires pour la synthèse des recommandations. Toute maladie associée au VRS doit faire l'objet d'une définition cohérente et précise afin de pouvoir comparer l'efficacité potentielle et l'efficacité réelle des différents candidats vaccins contre le VRS.

III. Protection contre les souches VRS/A et VRS/B. Les souches VRS/A et VRS/B sont typées sur leurs protéines de surface $G$. Les vaccins pourraient avoir une efficacité différentielle sur ces souches. Les sites antigéniques ciblés par certains candidats vaccins contre le VRS sont situés sur la protéine de surface $F$, en particulier les sites antigéniques $\varnothing$ et $V$ qui diffèrent entre les souches $A$ et $B(7)$. Des recherches complémentaires sont nécessaires pour confirmer l'efficacité de la protection apportée par ces vaccins contre les deux souches du VRS.

IV. Effet d'une immunité préexistante contre l'adénovirus sur l'efficacité des vaccins. Plusieurs candidats vaccins contre le VRS utilisent des adénovirus de l'homme ou du chimpanzé comme vecteurs pour libérer les antigènes du VRS. Ces vecteurs peuvent déclencher une réaction immunitaire spécifique susceptible d'atténuer ou d'accroître la réaction immunitaire aux antigènes du VRS, de compromettre I'efficacité du vaccin ou de modifier le profil d'innocuité du vaccin chez des personnes jusque-là immunisées. Des données probantes sont nécessaires pour déterminer la prévalence et l'effet de l'immunité contre l'adénovirus.
V. Effet des mutations d'échappement potentielles. Le palivizumab cible le site conservé II sur la protéine $\mathrm{F}$ du VRS. Le nirsevimab, un nouvel anticorps monoclonal développé par AstraZeneca/Sanofi Pasteur et actuellement soumis à des essais cliniques de phase 3 , cible le site $\varnothing$ sur la protéine F du VRS (4). Dans des conditions de pression sélective, les variantes virales pourraient changer la présentation de ces sites antigéniques (en modifiant la séquence de protéines ou les motifs de glycosylation) au point où ces anticorps monoclonaux cesseraient d'être efficaces. Des mécanismes de surveillance doivent être mis en place pour détecter les mutants d'échappement dans les infections survenant chez les personnes traitées par anticorps monoclonaux.

\section{Résultats clés : surveillance de la santé publique \\ Concordance entre la surveillance et les priorités de santé publique}

Les données de surveillance sur lesquelles se fondent la plupart des PSP sont accessibles auprès de deux sources au Canada : le Programme canadien de surveillance active de l'immunisation (IMPACT) (8), un système de surveillance sentinelle en milieu hospitalier, et la Base de données sur la morbidité hospitalière de I'Institut canadien d'information sur la santé (ICIS) (9), une base de données administratives hospitalières. Le tableau 2 illustre la enationale ou les sources d'information fournissent des données

Tableau 2 : Disponibilité des données de surveillance nécessaires pour évaluer l'efficacité du vaccin contre le VRS et répondre aux priorités de santé publique par groupe cible

\begin{tabular}{|c|c|c|c|c|c|}
\hline \multirow{3}{*}{$\begin{array}{l}\text { Priorité de santé } \\
\text { publique }\end{array}$} & \multicolumn{5}{|c|}{$\begin{array}{l}\text { Paramètres de santé publique } \\
\text { Mesures employées par le CCNI pour évaluer le } \\
\text { fardeau de morbidité }\end{array}$} \\
\hline & \multirow{2}{*}{$\begin{array}{l}\text { Incidence } \\
\text { IMPACT }^{c} \\
\text { ICIS }^{c}\end{array}$} & \multirow{2}{*}{$\begin{array}{c}\begin{array}{c}\text { Cadre } \\
\text { de }\end{array} \\
\text { l'étude } \\
\text { IMPACT }^{\mathrm{c}} \\
\text { ICIS }^{\mathrm{c}}\end{array}$} & \multirow{2}{*}{$\begin{array}{l}\text { Souche } \\
\text { du virus }\end{array}$} & \multicolumn{2}{|c|}{$\begin{array}{l}\text { Populations } \\
\text { présentant un } \\
\text { risque élevéb }\end{array}$} \\
\hline & & & & IMPACT $^{d}$ & $\mathrm{ICIS}^{\mathrm{c}}$ \\
\hline $\begin{array}{l}\text { Décès des } \\
\text { nourrissons }\end{array}$ & $\begin{array}{l}\mathrm{IMPACT}^{\mathrm{C}} \\
\mathrm{ICIS}^{\mathrm{c}}\end{array}$ & $\begin{array}{l}\text { IMPACT }^{c} \\
\text { ICIS }^{c}\end{array}$ & IMPACT $^{d}$ & IMPACT $^{d}$ & $\mathrm{ICIS}^{\mathrm{c}}$ \\
\hline $\begin{array}{l}\text { Infection dans } \\
\text { les collectivités } \\
\text { éloignées (enfant) }\end{array}$ & $\begin{array}{l}\text { IMPACT } \\
\text { ICIS }^{c}\end{array}$ & $\begin{array}{l}\text { IMPACT }^{\mathrm{C}} \\
\text { ICIS }^{\mathrm{C}}\end{array}$ & $\mathrm{IMPACT}^{\mathrm{C}}$ & \multicolumn{2}{|c|}{$\begin{array}{l}\mathrm{IMPACT}^{\mathrm{C}} \\
\mathrm{ICIS}^{\mathrm{c}}\end{array}$} \\
\hline $\begin{array}{l}\text { Hospitalisation des } \\
\text { enfants }\end{array}$ & $\begin{array}{l}\text { IMPACT }^{c} \\
\text { ICIS }^{c}\end{array}$ & $\begin{array}{l}\text { IMPACT } \\
\text { ICIS }^{c}\end{array}$ & IMPACT $^{d}$ & IMPACT $^{d}$ & $\mathrm{ICIS}^{\mathrm{c}}$ \\
\hline Décès des enfants & $\begin{array}{l}\text { IMPACTC }^{c} \\
\text { ICIS }^{c}\end{array}$ & $\begin{array}{l}\text { IMPACT }^{\mathrm{IMPA}} \\
\text { ICIS }^{\mathrm{C}}\end{array}$ & IMPACT ${ }^{d}$ & IMPACT $^{d}$ & $\mathrm{ICIS}^{\mathrm{c}}$ \\
\hline $\begin{array}{l}\text { Hospitalisation des } \\
\text { personnes âgées }\end{array}$ & $\mathrm{ICIS}^{\mathrm{c}}$ & $\mathrm{ICIS}^{\mathrm{c}}$ & $\mathrm{PD}^{\mathrm{e}}$ & \multicolumn{2}{|c|}{$\mathrm{ICIS}^{\mathrm{c}}$} \\
\hline $\begin{array}{l}\text { Décès des personnes } \\
\text { âgées }\end{array}$ & $\mathrm{ICIS}^{\mathrm{c}}$ & $\mathrm{ICIS}^{\mathrm{c}}$ & $P D^{e}$ & \multicolumn{2}{|c|}{$\mathrm{ICIS}^{\mathrm{c}}$} \\
\hline $\begin{array}{l}\text { Abréviations : CCNI, Com } \\
\text { d'information sur la santé, } \\
\text { PD, pas de données; VRS, } \\
\text { L'étude a pu être menée } \\
\text { primaires } \\
\text { Les populations qui prés } \\
\text { hourrissons, les enfants et } \\
\text { Données limitées - jaune } \\
\text { Données suffisantes - gri } \\
\text { Pas de données - rose }\end{array}$ & $\begin{array}{l}\text { is respirato } \\
\text { ss un cadre } \\
\text { ent un risqu } \\
\text { personnes }\end{array}$ & $\begin{array}{l}\text { cytial } \\
\text { hunautai }\end{array}$ & P YPC & 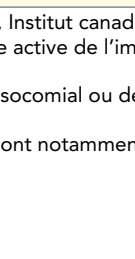 & oins \\
\hline
\end{tabular}


probantes à l'appui des PSP définies par les PT concernant le VRS.

Trois experts en surveillance de l'ASPC ont réalisé conjointement une évaluation subjective de chaque système ou source d'information pour déterminer si les données à l'appui du processus décisionnel étaient suffisantes, limitées ou inexistantes. IMPACT a été en mesure de fournir suffisamment de données sur les caractéristiques des souches virales et les populations pédiatriques présentant un risque élevé (nourrissons et enfants hospitalisés dans les centres IMPACT à cause du VRS). IMPACT a été en mesure de fournir des données limitées sur les taux d'incidence et d'infection dans les collectivités éloignées, mais aucune donnée sur les maladies associées au VRS ne nécessitant pas d'hospitalisation. Les taux d'incidence sont difficiles à calculer pour certains centres, car les circonscriptions hospitalières des centres ne concordent pas avec les statistiques démographiques (se reporter au tableau 3 pour de plus amples renseignements). Comme il n'existe aucun centre IMPACT dans les trois territoires et dans les régions nordiques des provinces touchées, le seul moyen de comptabiliser les cas de VRS existant dans ces collectivités serait de transférer les patients vers un hôpital IMPACT. Le cadre de l'étude se limite aux salles d'hôpital et aux services de soins intensifs.

\section{Tableau 3 : Survol des lacunes de surveillance relevées durant l'évaluation des données disponibles sur les populations prioritaires}

\begin{tabular}{|c|c|c|}
\hline $\begin{array}{l}\text { Population } \\
\text { prioritaire }\end{array}$ & $\begin{array}{l}\text { Lacune dans les } \\
\text { données }\end{array}$ & $\begin{array}{l}\text { Stratégie préconisée pour } \\
\text { combler la lacune }\end{array}$ \\
\hline \multirow[b]{2}{*}{$\begin{array}{l}\text { Cas de moins } \\
\text { de } 17 \text { ans }\end{array}$} & $\begin{array}{l}\text { Données utilisées } \\
\text { en guise de } \\
\text { dénominateur }\end{array}$ & $\begin{array}{l}\text { Mise en relation, à l'échelle des } \\
\text { hôpitaux, des centres IMPACT } \\
\text { avec I'ICIS }\end{array}$ \\
\hline & $\begin{array}{l}\text { Données sur les } \\
\text { cas d'infection } \\
\text { par le VRS sans } \\
\text { prise en charge } \\
\text { médicale }\end{array}$ & Aucune proposition \\
\hline $\begin{array}{l}\text { Cas présents } \\
\text { dans les } \\
\text { collectivités } \\
\text { autochtones } \\
\text { et éloignées }\end{array}$ & $\begin{array}{l}\text { Données sur } \\
\text { l'incidence, la } \\
\text { prévalence ou sur } \\
\text { certains facteurs } \\
\text { de risque }\end{array}$ & $\begin{array}{l}\text { Des projets pilotes de } \\
\text { surveillance permettraient de } \\
\text { déterminer plus précisément le } \\
\text { fardeau de morbidité ainsi que } \\
\text { l'acceptabilité et la faisabilité } \\
\text { des programmes de vaccination } \\
\text { dans ces collectivités à risque }\end{array}$ \\
\hline $\begin{array}{l}\text { Cas parmi les } \\
\text { personnes } \\
\text { âgées }\end{array}$ & $\begin{array}{l}\text { Données sur } \\
\text { l'incidence, la } \\
\text { prévalence, la } \\
\text { souche ou sur } \\
\text { certains facteurs } \\
\text { de risque }\end{array}$ & $\begin{array}{l}\text { Étude de cohorte rétrospective } \\
\text { des cas de VRS confirmés en } \\
\text { laboratoire par l'entremise du } \\
\text { réseau CIRN-SOS } \\
\text { Surveillance prospective par } \\
\text { l'entremise d'un réseau de } \\
\text { surveillance sentinelle }\end{array}$ \\
\hline
\end{tabular}

Abréviations : CIRN-SOS, Réseau de surveillance des cas sévères du Réseau canadien de recherche sur l'immunisation; ICIS, Institut canadien d'information sur la santé; IMPACT, Programme canadien de surveillance active de l'immunisation; VRS, virus respiratoire syncytial

L'ICIS a été en mesure de fournir des données limitées sur les hospitalisations pour toutes les classes d'âge et tous les paramètres de santé publique, à l'exception des caractéristiques des souches virales. La disponibilité des données obtenues par l'entremise de I'ICIS a été jugée limitée, car les hôpitaux de soins actifs (9) ne procèdent pas systématiquement à l'évaluation virologique, et une modélisation est nécessaire pour estimer le nombre d'hospitalisations imputables au VRS. Des données sur les populations présentant un risque élevé sont également disponibles auprès de I'ICIS, mais elles sont limitées par la manière dont les comorbidités sont consignées dans les bases de données administratives. Le champ du diagnostic principal et 24 autres champs connexes peuvent être analysés pour rechercher les comorbidités, mais leur utilisation varie en fonction des besoins cliniques.

\section{Lacunes dans les données relatives à trois populations prioritaires}

Les participants à la retraite ont cerné trois populations prioritaires pour lesquelles un plus grand nombre de données étaient nécessaires (se reporter au tableau 3).

I. Personnes de moins de 17 ans :

a. Absence de données utilisées en guise de dénominateur - Le système IMPACT vise à fournir des données sur I'hospitalisation des enfants de 16 ans et moins en lien avec le VRS. II s'agit néanmoins d'un système de surveillance sentinelle (et non fondé sur l'ensemble de la population), et les circonscriptions hospitalières de certains centres ne concordent pas avec les données démographiques disponibles. C'est pourquoi les données issues de ce système sont, à l'heure actuelle, limitées par le nombre de phénomènes de santé (p. ex. nombre d'admissions, nombre de décès, nombre d'admissions en service de soins intensifs). Interpréter ces indicateurs sans connaître le contexte de la population à laquelle ils se rattachent est une tâche complexe qui pourrait être simplifiée par le calcul des indicateurs démographiques. La mise en relation, à l'échelle des hôpitaux, des centres IMPACT avec les données administratives hospitalières de I'ICIS pourrait être envisagée pour faciliter la validation des tendances, comparer le nombre de patients comptabilisés par les différentes sources de données, examiner la représentativité et l'exhaustivité du réseau sentinelle et confirmer les données utilisées en guise de dénominateur.

b. Absence de données sur les cas d'infection par le VRS sans prise en charge médicale - II n'existe aucune donnée sur de tels cas d'infection par le VRS sans prise en charge médicale, données par ailleurs nécessaires à la modélisation de la transmission du VRS et à la réalisation d'études de coût-efficacité. Aucune stratégie n'a été préconisée durant la retraite pour combler cette lacune.

II. Collectivités autochtones et éloignées - Les collectivités autochtones et éloignées se heurtent à des obstacles supplémentaires dans l'accès aux soins de santé et souffrent d'une sous-représentation dans les systèmes actuels de surveillance nationale. Les estimations sur l'incidence, la prévalence ou certains facteurs de risque ne sont pas systématiquement compilées pour les collectivités 
autochtones. Le système IMPACT comptabilise les patients des collectivités autochtones et éloignées qui sont hospitalisés ou transférés dans un centre IMPACT, mais ces données ne reflètent pas le véritable fardeau de morbidité qui touche cette population. Des projets pilotes de surveillance sont en cours dans certaines collectivités autochtones éloignées du Québec afin de déterminer le fardeau de morbidité ainsi que l'acceptabilité et la faisabilité des programmes de vaccination (6). L'évolution que connaît le secteur des produits de vaccination proposés dans la prévention du VRS est l'occasion de voir apparaître des produits d'une plus grande longévité et durabilité. Des études complémentaires sont nécessaires pour évaluer les besoins des collectivités autochtones et éloignées.

III. Personnes âgées - Les données nationales sur les cas d'infection par le VRS chez les personnes âgées se limitent aux données administratives hospitalières de l'ICIS. Les données probantes laissent penser que les taux et nombres bruts de cas de VRS recueillis à partir cette source sousestiment le fardeau de morbidité chez les personnes âgées en raison du caractère incomplet de l'évaluation virologique dans ce groupe d'âge (10). Malgré les limites induites par les données manquantes, les données administratives demeurent une importante source de données. La collecte de données primaires sur l'épidémiologie et le fardeau de morbidité est justifiée pour les évaluations chez les personnes âgées. Deux stratégies visant à répondre à ce besoin ont été proposées lors des échanges :

a. Une étude de cohorte rétrospective des cas de VRS confirmés en laboratoire chez les patients de 65 ans et plus hospitalisés pour des syndromes grippaux est actuellement menée par l'entremise du réseau de surveillance des cas sévères du Réseau canadien de recherche sur l'immunisation (11). La possibilité que les données issues de cette étude puissent combler les lacunes existantes dans les données relatives aux personnes âgées pourrait être étudiée.

b. La faisabilité de la mise en place d'une surveillance prospective du VRS chez les personnes âgées par l'entremise d'un réseau de surveillance sentinelle pourrait être étudiée.

Les autres limites des systèmes de surveillance ont été examinées :

- Comprendre les séquelles à long terme du VRS permet d'élaborer des programmes de vaccination et des modèles économiques. Les données actuelles sur le rôle du VRS dans l'apparition de la bronchopneumopathie chronique obstructive ou de l'asthme sont peu concluantes. Des études complémentaires sont nécessaires pour mieux comprendre ce lien et le fardeau de morbidité du VRS sur le long terme.
- Certains systèmes de surveillance des infections respiratoires et du VRS utilisent les définitions de cas des syndromes grippaux pour définir leurs populations cibles. Les raisons qui les y amènent sont souvent de nature opportuniste (les systèmes de surveillance du VRS s'appuient sur I'infrastructure existante de surveillance de la grippe et sur les définitions des syndromes grippaux pour détecter les cas suspects) ou pratique (le processus de sélection des personnes pour le prélèvement des échantillons repose sur des algorithmes d'essais cliniques existants ou sur des normes de soins). L'inclusion de la fièvre dans la définition de cas du syndrome grippal peut amener à omettre des cas de VRS. II n'existe néanmoins aucune définition de cas normalisée des syndromes de l'infection par le VRS. Pour s'attaquer à ce problème, I'ASPC a participé à une collaboration sous l'égide de l'Organisation mondiale de la santé dans le but d'élaborer une définition de cas pour la surveillance du VRS, définition qu'elle ne manquera pas d'appliquer à ses initiatives de surveillance ou à ses analyses (12).

- Les systèmes actuels de surveillance reposent sur la surveillance passive des personnes qui demandent des soins médicaux en lien avec le VRS. Pour estimer le fardeau de morbidité du VRS sans prise en charge médicale, il faudrait mettre en place une stratégie active de surveillance. Bien qu'une telle approche soit complexe à réaliser, les données qui en résulteraient sont indispensables à l'élaboration d'un modèle de transmission du VRS et d'un modèle économique exacts et précis.

\section{Forces et faiblesses}

Bien que l'analyse ci-dessus représente le raisonnement des experts pour justifier la réalisation de nouvelles études et l'exécution de stratégies destinées à combler les lacunes, certaines réserves méritent d'être émises à l'égard de notre approche. Tout d'abord, l'analyse est limitée à l'expertise des participants. Les personnes invitées n'étaient pas toutes présentes en raison des contraintes liées à l'organisation d'une table ronde en personne. De ce fait, ces écrits ne constituent pas une analyse exhaustive du domaine concerné.

Ensuite, les PSP déterminées en collaboration avec les PT représentent un large éventail de besoins régionaux. Bien qu'un consensus se soit dégagé en amont de la retraite au sujet des priorités retenues, nous reconnaissons que celles-ci ne représentent pas chaque collectivité, province ou territoire, et que cette analyse est à considérer comme une estimation approximative plutôt que comme un tableau précis.

Enfin, le secteur du développement de vaccins et d'anticorps monoclonaux contre le VRS évolue à une telle vitesse que ce compte rendu sera caduc avant même d'être publié. Toutefois, alors même que des candidats vaccins sont en cours de développement, la question de la concordance avec les PSP et de la détermination des lacunes dans les données incitera le secteur à produire des vaccins de plus en plus efficaces. 


\section{Conclusion}

Cette retraite a montré la faisabilité d'un débat sur les vaccins potentiels et mis en évidence l'intérêt des intervenants à égard d'un tel débat. Elle a apporté un éclairage précieux sur les paramètres de santé publique importants dans la prévention du VRS, les mécanismes de surveillance actuellement employés et les questions qui demeurent en suspens.

\section{Déclaration des auteurs}

A. K. - Rédaction de la première version, administration du projet, conceptualisation

A. S. - Rédaction, révision, édition, conceptualisation et supervision

M. W. Y. - Rédaction, révision, édition et conceptualisation

M. T. - Rédaction, révision, édition, conceptualisation et supervision

C. B. - Rédaction, révision, édition, conceptualisation et supervision

A. H. - Rédaction, révision, édition, conceptualisation et supervision

W. V. - Rédaction, révision, édition, conceptualisation et supervision

D. M. - Rédaction, révision, édition, conceptualisation et supervision

C. Q. - Rédaction, révision, édition, conceptualisation et supervision

\section{Conflit d'intérêts}

Aucun.

\section{Remerciements}

Ce document est un résumé des échanges et des analyses des nombreux participants à la retraite de préparation à l'introduction des vaccins contre le VRS, notamment N. Crowcroft, G. De Serres, N. Gnidziejko, J. Langley, J. LeBlanc, M. Naus, J. Papenburg, E. Rafferty et G. Poliquin. Nous tenons à les remercier chaleureusement pour leurs contributions essentielles à cette analyse. Les auteurs tiennent également à reconnaître la contribution importante de S. Sandhu qui s'est attaché, avant et pendant la retraite, à étayer le contexte de surveillance du VRS et l'analyse des lacunes.

\section{Financement}

L'Agence de la santé publique du Canada a organisé la rencontre sur place et pris en charge les frais de déplacement des participants.

\section{Références}

1. Erickson LJ, De Wals P, Farand L. An analytical framework for immunization programs in Canada. Vaccine 2005 Mar;23(19):2470-6. DOI PubMed

2. Shi T, McAllister DA, $\mathrm{O}^{\prime}$ Brien $\mathrm{KL}$, Simoes EA, Madhi SA, Gessner BD, Polack FP, Balsells E, Acacio S, Ag uayo $C$, Alassani I, Ali A, Antonio M, Awasthi S, Awori JO, Azziz-Baumgartner E, Baggett HC, Baillie VL, Balmased A, Barahona A, Basnet S, Bassat Q, Basualdo W, Bigogo G, Bont L, Breiman RF, Brooks WA, Broor S, Bruce N, Bruden D, Buchy P, Campbell S, Carosone-Link P, Chadha M, Chipeta J, Chou M, Clara W, Cohen C, de Cuellar E, Dang DA, Dash-Yandag B, Deloria-Knoll M, Dherani M, Eap T, Ebruke BE, Echavarria M, de Freitas Lázaro Emediato CC, Fasce RA, Feikin DR, Feng L, Gentile A, Gordon A, Goswami D, Goyet S, Groome M, Halasa N, Hirve S, Homaira N, Howie SR, Jara J, Jroundi I, Kartasasmita CB, Khuri-Bulos N, Kotloff KL, Krishnan A, Libster R, Lopez O, Lucero MG, Lucion F, Lupisan SP, Marcone DN, McCracken JP, Mejia M, Moisi JC, Montgomery JM, Moore DP, Moraleda C, Moyes J, Munywoki P, Mutyara K, Nicol MP, Nokes DJ, Nymadawa P, da Costa Oliveira MT, Oshitani H, Pandey N, Paranhos-Baccalà G, Phillips LN, Picot VS, Rahman M, Rakoto-Andrianarivelo M, Rasmussen ZA, Rath BA, Robinson A, Romero C, Russomando G, Salimi V, Sawatwong P, Scheltema N, Schweiger B, Scott JA, Seidenberg P, Shen K, Singleton R, Sotomayor V, Strand TA, Sutanto A, Sylla M, Tapia MD, Thamthitiwat S, Thomas ED, Tokarz R, Turner C, Venter M, Waicharoen S, Wang J, Watthanaworawit W, Yoshida LM, Yu H, Zar HJ, Campbell H, Nair H; RSV Global Epidemiology Network. Global, regional, and national disease burden estimates of acute lower respiratory infections due to respiratory syncytial virus in young children in 2015: a systematic review and modelling study. Lancet 2017 Sep;390(10098):946-58. DOl PubMed

3. Shi T, Denouel A, Tietjen AK, Campbell I, Moran E, Li X, Campbell H, Demont C, Nya-wanda BO, Chu HY, Stoszek SK, Krishnan A, Openshaw P, Falsey AR, Nair H; RESCEU Investigators. RESCEU Investigators. Global disease burden estimates of respiratory syn-cytial virus-associated acute respiratory infection in older adults in 2015: a systematic re-view and meta-analysis. J Infect Dis 2019 Mar:jiz059. DOI PubMed

4. Killikelly A, Tunis M, House A, Quach C, Vaudry W, Moore $D$. Aperçu de la filière de candidats vaccins contre le virus respiratoire syncytial au Canada. Relevé des maladies transmissible au Canada 2020;46(04):63-70. DOI

5. Wilson SE, Rosella LC, Wang J, Le Saux N, Crowcroft NS, Harris T, Bolotin S, Deeks SL. Population-level impact of Ontario's infant rotavirus immunization program: evidence of direct and indirect effects. PLoS One 2016 May;11(5):e0154340. DOI PubMed 
6. Gilca R, Billard M, Lorcy A, Rochette M, Papenburg J, Zafack J, Charest H, Dionne M, Boucher F, De Serres G. Evaluation of new palivizumab immunoprophylaxis recommenda-tions in Nunavik infants: results for 2014 to 2017. Quebec City (QC): Bibliothèque et Ar-chives nationales du Québec: Institut national de santé publique Québec (INSPQ); 2018. https://nrbhss.ca/sites/default/files/documentations/report_ palivizumab_immunoprophylaxis_nunavik_infants.pdf

7. Hause AM, Henke DM, Avadhanula V, Shaw CA, Tapia LI, Piedra PA. Sequence variabil-ity of the respiratory syncytial virus (RSV) fusion gene among contemporary and historical genotypes of RSV/A and RSV/B. PLoS One 2017 Apr;12(4):e0175792. DOl PubMed

8. Programme canadien de surveillance active de I'immunisation (IMPACT). Ottawa (ON) : Société canadienne de pédiatrie; 2019 Dec 4 (Accédé 2019-12-20). https://www. cps.ca/fr/impact
9. Base de données sur la morbidité hospitalière. Ottawa $(\mathrm{ON})$ : Institut canadien d'information sur la santé; 2019 Nov (Accédé 2019-12-20). https://www.ICIS.ca/fr/ base-de-donnees-sur-la-morbidite-hospitaliere

10. Schanzer DL, Saboui M, Lee L, Nwosu A, Bancej C. Burden of influenza, respiratory syn-cytial virus, and other respiratory viruses and the completeness of respiratory viral identi-fication among respiratory inpatients, Canada, 20032014. Influenza Other Respir Viruses 2018 Jan;12(1):113-21. DOI PubMed

11. Serious outcomes surveillance (SOS) network. Halifax (NS): CIRN; 2019 (Accédé 2019-12-20). http://cirnetwork.ca/ network/serious-outcomes/

12. WHO global RSV surveillance pilot - objectives. Geneva $(\mathrm{CH})$ : World Health Organization; (Accédé 2019-12-20). https:// www.who.int/influenza/rsv/rsv_objectives/en/

\section{Annexe 1 : Liste des participants à la retraite de préparation à l'introduction des vaccins contre le VRS}

\section{Nom}

Caroline Quach

Sarah Buchan

Natasha Crowcroft

Gaston De Serres

Shelley Deeks

Nick Gnidziejko

Joanne Langley

Jason LeBlanc

Dorothy Moore

Monika Naus

Jesse Papenburg

Ellen Rafferty

Beate Sander

Wendy Vaudry

Christina Bancej

Erin Henry

Althea House

April Killikelly

Robert Nesdole

Linda Pelude

Robert Pless

Guillaume Poliquin

Simran Sandhu

Amanda Shane

Veeran-Anne Singh

Matthew Tunis

Man Wah Yeung
Affiliation professionnelle

Université de Montréal, Centre hospitalier universitaire (CHU) Sainte-Justine

Santé Publique Ontario

Santé Publique Ontario

Institut national de santé publique du Québec (INSPQ)

Santé Publique Ontario

Institut canadien d'information sur la santé (ICIS)

Université Dalhousie et Réseau canadien de recherche sur l'immunisation (RCRI)

Régie de la santé de la Nouvelle-Écosse

Université McGill

Centre de contrôle des maladies de la Colombie-Britannique (BCCDC)

Université McGill

Université d'Alberta

Toronto Health Economics and Technology Assessment (THETA)

Université d'Alberta

Centre de l'immunisation et des maladies respiratoires infectieuses (CIMRI), Division de surveillance de la santé et de

l'épidémiologie (DSE)

CIMRI

CMRI, Secrétariat du Comité consultatif national de l'immunisation (CCNI)

CIMRI, Secretariat du CCNI

Direction générale de la santé des Premières nations et des Inuits (DGSPNI)

Centre de la lutte contre les maladies transmissibles et les infections (CLMTI), Programme canadien de surveillance des infections nosocomiales (PCSIN)

Santé Canada, Direction des produits biologiques et des thérapies génétiques (DPBTG)

Laboratoire national de microbiologie (LNM)

CIMRI, DSE

CIMRI, DSE

DGSPNI

CIMRI, Secrétariat du CCNI

CIMRI, Secrétariat du CCNI 